\title{
O trabalho do Agente Comunitário de Saúde
}

The work of the community health agent

Ana Cláudia Garabeli Cavalli Kluthcovsky ${ }^{1}$

Angela Maria Magosso Takayanagui ${ }^{2}$

\section{RESUMO}

Este artigo tem como objetivo refletir sobre o Agente Comunitário de Saúde (ACS) inserido em um contexto de desafios, pela necessidade de mudança no modelo de atenção à saúde. Historicamente, representa o elo entre o sistema de saúde e a comunidade onde vive e trabalha, sendo, por isso, um trabalhador singular no âmbito da saúde. O estudo envolve metodologia descritivo-reflexiva, com dados fundamentados na literatura. Apresenta-se um breve histórico sobre a Estratégia Saúde da Família, enquanto proposta de reorganização da Atenção Básica, e questões referentes ao serviço do ACS, suas competências e o processo de trabalho. Foi possível perceber a importância desse profissional pelo número crescente de profissionais em atividade e pela relevância de seu trabalho na vigilância e promoção da saúde.

\section{ABSTRACT}

In this article we reflect about the Community Health Agent (CHA), a professional working in a context of challenges arising from the need for changing the health care model. Traditionally, this professional represents the link between the health system and the community where he/she lives and works, thus performing a singular activity in the health environment. The study adopts a descriptive-reflexive methodology, based on data in the literature. It presents a historical briefing about the Family Health Strategy as a strategy for reorganizing primary care and about questions regarding the work of the health agent, his competences and work process. It was possible to perceive the importance of this professional due to the increasing number of professionals in activity and due to the relevance of their work for health promotion and surveillance.

PALAVRAS-CHAVE:

- Programa Saúde da Família;

- Atenção Primária à Saúde.
KEY-WORDS:

- Family Health Program;

- Primary Health Care.

\footnotetext{
${ }^{1}$ Médica, Mestre em Saúde Pública, Professora, Departamento de Enfermagem, Universidade Estadual do Centro-Oeste, Brasil.

2 Enfermeira, Professora Associada, Departamento de Enfermagem Materno-Infantil e Saúde Pública, Escola de Enfermagem de Ribeirão Preto, Universidade de São Paulo, Ribeirão Preto, Brasil.
} 


\section{Introdução}

O complexo processo de construção do Sistema Único de Saúde (SUS) envolve ações de mudança na gestão, na organização e no financiamento do sistema ${ }^{1}$, buscando a mudança do modelo assistencial e desenvolvendo ações de saúde caracterizadas pelas resolutividade e integralidade ${ }^{2}$.

Nesse processo, a reestruturação da Atenção Primária à Saúde (APS) constitui-se um nó crítico, e a Estratégia Saúde da Família (ESF) tornou-se uma política de âmbito nacional ${ }^{3}$, como proposta do Ministério da Saúde (MS) para esta reestruturação.

Uma equipe de saúde da família deve ser formada, no mínimo, por: um médico generalista ou de família; um enfermeiro; um ou dois auxiliares de enfermagem e Agentes Comunitários de Saúde (ACS). Além desses, outros profissionais de nível superior têm sido integrados às equipes, pela necessidade de enfoque multiprofissional e interdisciplinar.

No tocante à importância da equipe de saúde para a ESF, há de se fazer menção ao Agente Comunitário de Saúde, profissional cujas diretrizes funcionais foram fixadas pela Portaria ํㅡㄴ.189 de 1999, na qual Ihes é atribuída a função de: “[...] desenvolver atividades de prevenção de doenças e promoção de saúde, por meio de ações educativas individuais e coletivas, nos domicílios e na comunidade, na sua área de atuação" ${ }^{4}$.

Entendendo-se a relevância dessas reflexões sobre a saúde pública no Brasil, este estudo justifica-se, em especial, pela importância dos ACS na viabilização da ESF, no contexto do SUS.
Assim, este trabalho tem por objetivo fazer uma reflexão sobre o ACS inserido em um contexto de desafios pela necessidade de mudança no modelo de atenção à saúde. Historicamente, representa o elo entre o sistema de saúde e a comunidade onde vive e trabalha, sendo, por isso, um trabalhador singular em saúde. O estudo envolve metodologia descritivo-reflexiva, com dados fundamentados na literatura. São apresentados: um breve histórico sobre a ESF; questões referentes ao trabalho do ACS; além de suas competências e o processo de trabalho.

\section{A Estratégia Saúde da Família}

A ESF foi influenciada por diferentes modelos advindos de outros países. Suas origens diretas, porém, estão no Programa de Agentes de Saúde, instituído pela Secretaria Estadual de Saúde do Ceará, em 1987. Este modelo obteve muitos resultados positivos, sendo então estendido a todo o país, em 1991, pelo MS, como Programa de Agentes Comunitários de Saúde (PACS) ${ }^{3}$.

O PACS, por outro lado, constituiu uma proposta seletiva na APS, gerando uma demanda crescente nos serviços de saúde. Uma experiência pioneira surgiu para responder a essa demanda, em Quixadá, Ceará, e serviu de base para o Ministério da Saúde lançar, em 1994, o Programa Saúde da Família ${ }^{3}$.

O objetivo do Ministério da Saúde foi, a partir dessa iniciativa, realizar o pressuposto na Constituição Federal em relação aos princípios básicos do SUS, no que se refere à organização tradicional dos serviços de saúde, com uma proposta de mudança no paradigma de assistência pública à saúde. 
Em estudo sobre o PACS, observou-se que os profissionais reconhecem que o serviço de saúde organiza-se pela lógica das condições agudas, centrada na atenção curativa e individual, comprometendo as ações de prevenção e promoção à saúde 5 .

O principal problema a ser enfrentado resulta da reprodução do modelo assistencial privatista, que envolve relações entre o Estado, as empresas multinacionais de medicamentos e equipamentos médicos e o empresariado nacional e multinacional da área da saúde. Um outro desafio seria a implementação de uma política voltada à expansão e à qualificação da APS'.

É necessário que a equipe de saúde da família seja multiprofissional e conheça tanto as famílias como o ambiente do território de abrangência, para o desenvolvimento de ações educativas e intersetoriais, com prestação de assistência integral à saúde $e^{2,6,7}$.

Evidentemente, a ênfase nas ações de prevenção em saúde não pode e não deve excluir ou diminuir as ações curativas necessárias. Esta questão demonstra a necessidade de uma programação em saúde, como etapa do processo de planejamento, para que as metas sejam atingidas $^{2,8}$.

Trabalhos sobre a ESF, na literatura, têm revelado alguns resultados em indicadores de saúde, como em estudo realizado em Araraquara, São Paulo, de 1999 a 2002, no qual se observou o impacto, após a implantação da ESF, em cinco indicadores da atenção básica, com expressivas melhorias na saúde da população ${ }^{8}$.

Outro artigo relata a melhoria de uma série de indicadores de saúde, resultados das bases teóricas, da organização e do trabalho, durante oito anos de atividades das equipes saúde da família em Sobral, estado do Ceará ${ }^{\text {. }}$.

Em estudo sobre a ESF em Iguatu, Ceará, observou-se redução nos índices de mortalidade infantil, melhoria nos níveis de aleitamento materno, acompanhamento de $95 \%$ das gestantes cadastradas e índice de vacinação de 100\%. Alguns problemas citados foram: o excessivo número de famílias por equipe; a rotatividade dos médicos e a grande demanda por atendimentos em especialidades ${ }^{10}$.

Em uma avaliação qualitativa de satisfação de usuários em cinco municípios na Bahia, constatou-se um elevado grau de satisfação dos usuários quanto à estratégia, relacionado ao maior acesso aos cuidados médicos, melhoria do nível de informação em saúde e a existência da visita domiciliar. Dentre os aspectos negativos, destacaram-se a persistência das filas e a fragilidade no encaminhamento para os níveis secundário e terciário da atenção ${ }^{11}$.

Apesar da enorme expansão do número de equipes de saúde da família em todo o país, ainda não se pode afirmar que as ações e os serviços produzidos no conjunto reflitam uma mudança de conteúdo das práticas e da forma de organização do processo de trabalho, além da tendência à burocratização do processo de gerenciamento do trabalho das equipes ${ }^{1}$.

Sobre as dificuldades práticas, também é relatado que: "[...] tem-se verificado inúmeras dificuldades para transpor para o campo prático do processo de trabalho das equipes, as características transformadoras da Atenção Básica inerentes à proposta de saúde da família”2 
Inserido em todo este contexto de mudanças e desafios, que, até agora, vem apresentando resultados diversos, encontra-se o ACS, o qual pode integrar uma equipe de saúde da família ou fazer parte do PACS.

\section{O Agente Comunitário de Saúde}

Segundo dados do Departamento de Atenção Básica do Ministério da Saúde, em dezembro de 2005, o país contava com 210.887 ACS, sendo que $59 \%$ da população estimada do país contava com acompanhamento por ACS (base de cálculo: 575 pessoas por agente) e 45,3\%, pela ESF (base de cálculo: 3.450 pessoas por equipe $)^{12}$.

Os municípios, inicialmente, adotaram diversas formas de contratação dos ACS. Assim, muitas dessas formas não reconheciam os direitos trabalhistas previstos na Constituição Federal. Os ACS começaram, então, a reivindicar o reconhecimento da profissão, que foi estabelecido pela Lei $\mathrm{n}^{\circ} 10.507$, de 10 de julho de $2002^{13}$.

O Agente Comunitário de Saúde é um profissional sui generis, proveniente de sua comunidade, tendo que residir na própria área de trabalho há pelo menos dois anos, conhecedor da realidade local, sendo fundamentais os aspectos de solidariedade e liderança e que trabalha com adscrição de famílias em base geográfica definida. É responsável pelo acompanhamento de, no máximo, 750 pessoas $^{4,12,14}$.

Segundo o Ministério da Saúde, também são atribuições dos ACS: analisar as necessidades da comunidade; atuar nas ações de controle de doenças e promoção e proteção da saúde; participar das reuniões da equipe de saúde e da comunidade. Seus principais instrumentos de trabalho são: a entrevista, a visita domiciliar, o cadastramento das famílias, o mapeamento da comunidade e as reuniões comunitárias ${ }^{15}$.

O trabalho comunitário requer algumas características individuais dos prestadores de assistência, a começar pela percepção das potencialidades da comunidade, bem como dos problemas nela existentes, introduzindo novas alternativas, sem que, para isso, sejam negadas a cultura e a vivência dessa comunidade.

Historicamente, os ACS representam um elo entre a equipe profissional e a comunidade. Por conviver com a realidade do bairro onde mora e trabalha e ser formado a partir de referenciais biomédicos, o ACS torna-se um interlocutor das contradições e dos diálogos entre saberes e práticas, podendo ser um facilitador ou um empecilho nessa mediação ${ }^{7}$.

Os ACS também devem participar da importante questão da humanização dos serviços de saúde, que inclui o acolhimento do usuário nos serviços de saúde ${ }^{16}$.

Podem, além disso, enfrentar algumas situações particulares em seu trabalho, como as descritas em estudo realizado em cinco municípios no estado da Bahia. A pesquisa analisou as práticas e os discursos sobre os ACS, produzidos na realização de seu trabalho, por parte de usuários, informantes chaves e profissionais das equipes de saúde. Foram observados, em relação ao trabalho dos ACS: um aumento do seu poder de resolutividade; o papel de "tradutor" do universo científico ao popular; a entrada no mundo familiar das pessoas; ser facilitador do acesso das pespessoas da comunidade aos serviços de saúde; 
o reconhecimento do seu trabalho; um aumento do conhecimento biomédico; aumento de trabalho e de responsabilidades e, também, dificuldades de relacionamento com membros da comunidade?.

Devido a essas condições, os ACS podem sofrer, a cada dia, inúmeras situações de tensão, nas quais devem rever suas práticas diante de novos desafios. Por residirem nas áreas onde trabalham, vivem o cotidiano da comunidade, com seus aspectos positivos ou negativos, certamente com mais intensidade do que os outros membros da equipe de saúde.

Apesar das dificuldades encontradas na prática profissional, é inegável o benefício que o trabalho dos ACS em atividade no país tem proporcionado à saúde da população brasileira, pela contribuição na qualificação das ações de saúde, que resultam, por exemplo, em reduções dos índices de mortalidade infantil, aumento das taxas de cobertura pré-natal, de vacinação, de vigilância à saúde de mães, crianças, adolescentes, adultos e idosos ${ }^{17}$.

Estudo sobre o trabalho dos então chamados Agentes de Saúde do Ceará, iniciado em 1987, demonstrou redução da mortalidade infantil, aumento da utilização de soro de reidratação oral durante episódios de diarréia nos primeiros anos de vida e aumento da cobertura vacinal básica completa em crianças de 12 a 36 meses $^{18}$.

Outro exemplo do desempenho do trabalho dos ACS foi um estudo realizado sobre as mudanças em indicadores de saúde infantil ao longo de três anos, em 409 crianças, em média, em Itapirapuã Paulista, São Paulo, após a atuação de 12 ACS previamente treinados. Os indicadores de saúde infantil estudados foram sistemati- camente melhores que os indicadores de um município vizinho tomado como controle, revelando ser bastante provável que os ACS tenham contribuído para essas melhorias ${ }^{19}$.

Também a valorização da comunidade sobre o trabalho dos ACS tem sido estudada, estando relacionada, entre outros fatores, à diminuição da mortalidade infantil e de adultos na comunidade, à diminuição da incidência de diarréia e ao acompanhamento do desenvolvimento das crianças $^{20}$.

Em pesquisa realizada com famílias atendidas pelo PSF em áreas urbanas e rurais de cinco municípios do estado da Bahia, entre 1999 e 2001, o papel dos ACS também teve um julgamento positivo?.

Ainda há outros estudos que tratam dos aspectos relacionados à atuação dos ACS, de seu vínculo com a comunidade e da satisfação e do reconhecimento do seu trabalho por parte dos usuários $^{21,22}$.

\section{Considerações Finais}

A inserção dos ACS na APS no Brasil vem alcançando um destaque cada vez maior pelo importante papel que eles vêm desempenhando na ESF, revelando, em grande parte dos estudos, um impacto positivo nos resultados obtidos.

Considerando o grande número de ACS atuando no país, pode-se salientar que o seu trabalho é de extrema importância para o atual estágio da APS no Brasil, pois entram em contato com diferentes demandas diariamente, sendo, por esta razão, profissionais que merecem especial atenção.

Sem dúvida, o Agente Comunitário de 
Saúde, trabalhando como elo entre a equipe profissional e a comunidade, desenvolve ações em três dimensões: a técnica, operando com saberes da epidemiologia e clínica; a política, utilizando saberes da saúde coletiva, e a de assistência social, possibilitando o acesso com eqüidade aos serviços de saúde ${ }^{16}$ - o que lhe confere uma condição especial.

No entanto, apesar de todas essas atribuições, em alguns aspectos incongruentes, tratase, em geral, do grupo de menor escolaridade na equipe de saúde da família e, conseqüentemente, de pior remuneração. As tensões envolvidas no processo de trabalho e as respectivas ações e desempenho profissional certamente afetam a qualidade de vida do agente comunitário de saúde, o que, sem dúvida, merece estudos mais aprofundados, posto serem assuntos também relevantes para viabilização da APS e, por conseguinte, do próprio SUS.

\section{Referências}

1. Teixeira CF. Saúde da Família, promoção e vigilância: construindo a integralidade da atenção à saúde no SUS. Revista Brasileira de Saúde da Família. jan.2003-abr.2004; (7): 10-23.

2. Marcier MHFC. Para que a expansão do PSF seja consistente. Revista Brasileira de Saúde da Família. 2002; (6): 32-35.

3. Mendes EV. A evolução histórica da atenção primária à saúde no Brasil. In: Mendes EV. A Atenção Primária à Saúde no SUS. Fortaleza (CE): Escola de Saúde Pública do Ceará; 2002. p. 2329.

4. Brasil. Ministério da Saúde. Secretaria de Políticas de Saúde. Departamento de Atenção
Básica. Modalidade de Contratação de Agentes Comunitários de Saúde: Um Pacto Tripartite. Reimpressão. Brasília (DF): Ministério da Saúde; 2002. 43p.

5. Muniz JN. O Programa de Agentes Comunitários de Saúde na identificação de sintomáticos respiratórios no controle da tuberculose em Ribeirão Preto-SP: um desafio para a atenção primária à saúde [Tese de Doutorado]. Ribeirão Preto (SP): Escola de Enfermagem de Ribeirão Preto/USP; 2004.

6. Brasil. Ministério da Saúde. Secretaria de Políticas de Saúde. Departamento de Atenção Básica. Apresentação. Avaliação da implantação do Programa Saúde da Família em dez grandes centros urbanos: síntese dos principais resultados. Brasília: Ministério da Saúde; 2002. p. 13-20.

7. Nunes MO, Trad LB, Almeida BA, Homem CR, Melo MCIC. O agente comunitário de saúde: construção da identidade desse personagem híbrido e polifônico. Cadernos de Saúde Pública. 2002; 18(6): 1639-1646.

8. Mattos ATR, Caccia-Bava MCG. Repercussões da implantação do Programa Saúde da Família no município de Araraquara: impactos e perspectivas. Revista Brasileira de Medicina de Família e Comunidade. 2004; 1(1): 36-43.

9. Andrade LOM, Barreto ICHC, Goya N, Martins Júnior T. Estratégia Saúde da Família: oito anos construindo um modelo de atenção integral à saúde. SANARE. 2004; n.esp.(1): 9-20.

10. Franklin A. Iguatu: água grande, muita água, água boa. Revista Brasileira de Saúde da Família. 1999; (n.esp): 22-29.

11. Trad LAB, Bastos ACS, Santana EM, Nunes MO. Estudo etnográfico da satisfação do usuário 
do Programa de Saúde da Família (PSF) na Bahia. Ciência \& Saúde Coletiva. 2002; 7(3): 581-89.

12. Brasil. Ministério da Saúde. Departamento de Atenção Básica. Portal da Saúde. Agentes Comunitários de Saúde, Equipes de Saúde da Família e Equipes de Saúde Bucal, em atuação competência dezembro/2005. Disponível em: URL: http://dtr2004.saude.gov.br/dab/documentos/ resumo_por_uf-02_2006.pdf [2006 mar 30].

13. Lopes BC, Chagas DMNM, Torres ZF. Regulação profissional do Agente Comunitário de Saúde: natureza do trabalho, qualificação e vínculo. Revista Brasileira de Saúde da Família. 2002; (6): 28-31.

14. Conselho Nacional dos Secretários de Saúde - CONASS. Programa de informação e apoio técnico às novas equipes gestoras estaduais do SUS de 2003. Legislação do SUS. Brasília (DF): CONASS; 2003. 604 p.

15. Brasil. Ministério da Saúde. Secretaria de Políticas de Saúde. Departamento de Atenção Básica. Saúde e comunidade. O Trabalho do Agente Comunitário de Saúde. Brasília (DF): Ministério da Saúde; 2000. 119 p.

16. Chiesa AM, Fracolli LA. O trabalho dos Agentes Comunitários de Saúde nas grandes cidades: análise do seu potencial na perspectiva da Promoção da Saúde. Revista Brasileira de Saúde da Família. 2003-2004; 7(n.esp.): 42-49.

17. Fonseca CD. Apresentação. In: BRASIL. Ministério da Saúde. Secretaria de Políticas de Saúde. Departamento de Atenção Básica. Modalidade de Contratação de Agentes Comunitários de Saúde: Um Pacto Tripartite. Reimpressão. Brasília (DF): Ministério da Saúde; 2002. p. 5.
18. Svitone EC, Garfield R, Vasconcelos MI, Craveiro VA. Primary health care lessons from the Northeast of Brazil: the Agentes de Saúde Program. Revista Panamericana de Salud Publica. 2000; 7(5): 293-302.

19. César JA, Cavaleti MA, Holthausen RS, Lima LGS. Mudanças em indicadores de saúde infantil em um município com agentes comunitários: o caso de Itapirapuã Paulista, Vale do Ribeira, São Paulo, Brasil. Cadernos de Saúde Pública. 2002; 18(6): 1647-1654.

20. Pedrosa JIS, Teles JBM. Consenso e diferenças em equipes do Programa Saúde da Família. Revista de Saúde Pública. 2001; 35(3): 303-311. 21. Oliveira RG, Nachif MCA, Matheus MLF. O trabalho do agente comunitário de saúde na percepção da comunidade de Anastácio, Estado do Mato Grosso do Sul. Acta Scientiarum Health Sciences. 2003; 25(1): 95-101.

22. Levy FM, Matos PES, Tomita NE. Programa de agentes comunitários de saúde: a percepção de usuários e trabalhadores da saúde. Cadernos de Saúde Pública. 2004; 20(1): 197-203.

\section{Endereço para correspondência:}

Ana Cláudia Garabeli Cavalli Kluthcovsky

Universidade Estadual do Centro-Oeste CEDETEG

Departamento de Enfermagem

Rua Simeão Varela de Sá, 03, Vila Carli

CEP 85040-080, Guarapuava-PR

Endereço Eletrônico: anafabio@brturbo.com.br 